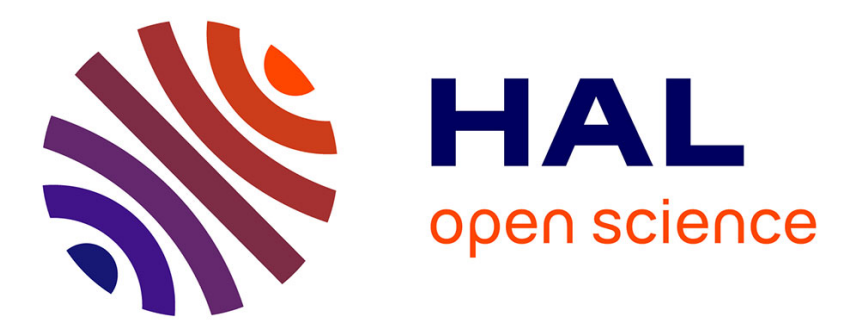

\title{
On the convergence of Boolean automata networks without negative cycles
}

\author{
Tarek Melliti, Damien Regnault, Adrien Richard, Sylvain Sené
}

\section{To cite this version:}

Tarek Melliti, Damien Regnault, Adrien Richard, Sylvain Sené. On the convergence of Boolean automata networks without negative cycles. 19th International Workshop on Cellular Automata and Discrete Complex Systems (AUTOMATA 2013), Sep 2013, Gießen, Germany. pp.124-138, 10.1007/9783-642-40867-0_9. hal-00832885

\section{HAL Id: hal-00832885 https://hal.science/hal-00832885}

Submitted on 28 Nov 2013

HAL is a multi-disciplinary open access archive for the deposit and dissemination of scientific research documents, whether they are published or not. The documents may come from teaching and research institutions in France or abroad, or from public or private research centers.
L'archive ouverte pluridisciplinaire HAL, est destinée au dépôt et à la diffusion de documents scientifiques de niveau recherche, publiés ou non, émanant des établissements d'enseignement et de recherche français ou étrangers, des laboratoires publics ou privés. 


\title{
On the convergence of Boolean automata networks without negative cycles
}

\author{
Tarek Melliti ${ }^{1}$, Damien Regnault ${ }^{1}$, Adrien Richard ${ }^{2}$, and Sylvain Sené ${ }^{1,3}$ \\ 1 Laboratoire IBISC, EA4526, Université d'Évry Val-d'Essonne, 91000 Évry, France \\ ( $\{$ tarek.melliti, damien.regnault, sylvain.sene\}@ibisc.univ-evry.fr) \\ 2 Laboratoire I3S, UMR7271 CNRS et Université de Nice Sophia Antipolis, 06900 \\ Sophia Antipolis, France \\ (richard@i3s.unice.fr) \\ 3 IXXI, Institut rhône-alpin des systèmes complexes, 69007 Lyon, France
}

\begin{abstract}
Since the 1980's, automata networks have been at the centre of numerous studies, from both theoretical (around the computational abilities) and applied (around the modelling power of real phenomena) standpoints. In this paper, basing ourselves on the seminal works of Robert and Thomas, we focus on a specific family of Boolean automata networks, those without negative cycles. For these networks, subjected to both asynchronous and elementary updating modes, we give new answers to well known problems (some of them having already been solved) about their convergence towards stable configurations. For the already solved ones, the proofs given are much simpler and neater than the existing ones. For the others, in any case, the proofs presented are constructive.
\end{abstract}

Keywords: Boolean automata networks, cycles, monotony, convergence and convergence time.

\section{Introduction}

Historically, the appearance of automata networks (ANs) in computer science flows from the works of McCulloch and Pitts on neural networks [14] and of von Neumann on cellular automata (CAs) [15], in the 1940's. In this way, they are amongst the first unconventional models of computation and constitute the origin of numerous key works in this domain, such as that of Kleene on finite automata [13] and of Elspas and Golomb on circuit theory [3,7]. Then, in the 1980 's, in a context at the frontier of discrete dynamical systems and models of computation, many researches were led on these objects that provided significant results on their dynamical behaviours and their expressiveness $[4,5$, 10,20]. Beyond these mostly theoretical works, the interest in ANs has been sustained through their application to biology. Since McCulloch and Pitts indeed, a thriving line of researches on ANs have been done about their ability to model biological regulation networks, with a special attention paid to genetic networks. Amongst the best representative studies in this domain are those of 
Kauffman [11] and Thomas [25], from which high impact results on some governing laws of living systems have been obtained (we will evoke some of them later). Relying on both these computational and biological standpoints, which contribute equally to the motivations of the paper, the study developed further focuses on the convergence of ANs.

Before we give more details about the contents of the paper, let us give precisions about ANs. From the most general point of view, an AN is a system of interacting computing units, the automata of the network, over a discrete time. Here, an automaton has to be considered as a discrete entity that computes a result, i.e. its updated state, according to a predetermined local transition function depending on the inputs it receives from other automata (possibly including itself) in the network, i.e. its neighbours. Thus, in some sense, an automaton can be viewed as a black box. The interactions between automata, that define the neighbourhood relations between them, are directed edges between automata. They can be of two sorts according to their activating ('+'-label) or inhibiting ('-'-label) nature. From this derives the fact that the architecture of an AN is captured by a digraph, classically called the interaction graph. Note that, in the specific context of CAs, the automata that are rather called cells share the same local transition function and are organised on a lattice graph. Let us highlight that, in this paper, we restrict ourselves to the study of finite ANs (i.e. of finite size, not to be confused with networks of finite automata). To go further, we speak of Boolean automata networks (BANs), meaning that automata states can only take Boolean values. From the computational point of view, it is well known that it is not a limitation [5,23]. However, from the modelling point of view where automata can represent genes for instance, it is. But it is deliberate in the sense that such a limitation allows to focus on the state changes rather than on the states themselves.

Now that the static aspect of BANs has been presented, let us add that their study is classically dived into a dynamical dimension. Being given an arbitrary BAN, with the concept of a configuration that corresponds to the attribution of a state to every automaton, its dynamical behaviour can be studied by executing its local transition functions over time. In general, the behaviour of a BAN is also represented by a digraph, called the transition graph (in the context of CAs, we rather speak of space-time diagram). In the sequel, we pay particular attention to transient and asymptotic behaviours of BANs. The asymptotic behaviours of a BAN, its attractors, are the terminal strongly connected components of its transition graph. Note that to focus on the dynamical aspect of a BAN asks for choosing an updating mode (e.g. a non-deterministic one [27], a deterministic one [20] or a probabilistic one [22]). In this work, we selected the (perfectly) asynchronous one, that gives non-deterministically from each configuration the ability of updating every automaton ${ }^{4}$.

Some peculiar architectural patterns of BANs, namely the cycles, are known to play a major role in their dynamical behaviours. Two kinds of cycles are

\footnotetext{
${ }^{4}$ Nevertheless, notice that the results extend naturally to the elementary updating mode [16], unless an explicit mention is given.
} 
distinguished: the positive and the negatives ones, the former being composed of an even number of negative arcs, the latter of an odd number of negative arcs. Let us now recall three results that constitute the basis of our study: (i) the Robert's theorem - if the interaction graph of a BAN does not contain any cycle, its dynamical behaviour is "trivial", i.e. it admits a unique attractor that is a stable configuration [20]; (ii) the first Thomas' rule - the presence of a positive cycle in a BAN is necessary for it to admit several stable configurations and (iii) the second Thomas' rule - the presence of a negative cycle is necessary for it to admit a stable oscillation [17-19,26]. From these, the general aim of this paper is to address the problem of the convergence (and the convergence time) of BANs with no negative cycles in their architecture. As a result, with $n$ denoting the number of automata (i.e. the size) of such a BAN, the main contributions are:

- a result showing that the absence of negative cycles in BANs implies Boolean monotonicity;

- a new proof of the second Thomas' rule, much simpler and neater;

- a result showing that, for any configuration, there exists a path of length at most $n$ in the transition graph from this configuration to a stable configuration.

In Section 2 are given the main definitions and notations used in the paper. Section 3 presents the results discussed above about the convergence of BANs with no negative cycles, before Section 4 concludes the paper and provides some relevant perspectives of this work.

\section{Definitions and notations}

This section gives the classical definitions and notations in the context of BANs. Those that are more specific will be given when they will prove to be useful.

\section{$2.1 \quad$ BANs}

Let $\mathbb{B}=\{0,1\}$ and let $\mathrm{V}=\{1, \ldots, n\}$ denote a set of $n \in \mathbb{N}$ Boolean automata such that $\forall i \in \mathrm{V}, x_{i} \in \mathbb{B}$ is the state of automaton $i$. A configuration of $\mathscr{N}$ is a vector $x \in \mathbb{B}^{n}$ that instantiates the state of each automaton of $\mathrm{V}$ (a configuration can be denoted by either a vector or a binary word). Because special attention is paid to automata switches in this paper, we introduce the following notations:

$$
\forall x \in \mathbb{B}^{n},
$$

1. $\forall i \in \mathrm{V}, \bar{x}^{i}=\left(x_{1}, \ldots, x_{i-1}, \neg x_{i}, x_{i+1}, \ldots, x_{n}\right)$, and

2. $\forall \mathrm{W}=\mathrm{W}^{\prime} \uplus\{i\} \subseteq \mathrm{V}, \bar{x}^{\mathrm{W}}={\overline{\left(\bar{x}^{i}\right)}}^{\mathrm{W}^{\prime}}={\overline{\left(\bar{x}^{\mathrm{W}}\right)}}^{i}$.

Furthermore, let $\mathbb{1}=\{-1,+1\}$ and $\mathrm{s}: \mathbb{B} \rightarrow \mathbb{1}$ be the function that switches from Boolean values to signed values such that $\mathrm{s}(b)=b-\neg b$. In order to compare two 


$$
\mathscr{N}_{1}=\left\{\begin{array}{l}
f_{1}(x)=x_{3} \\
f_{2}(x)=x_{1} \vee x_{3} \\
f_{3}(x)=x_{2} \wedge \neg x_{4} \\
f_{4}(x)=\neg x_{1}
\end{array}\right.
$$

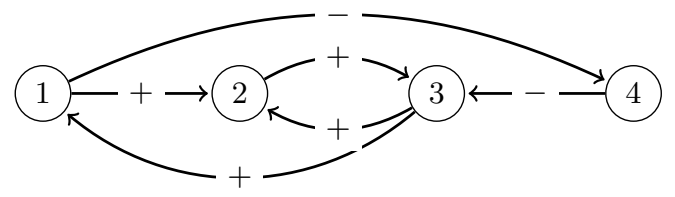

Fig. 1. A BAN $\mathscr{N}_{1}$ of size 4 and its signed interaction graph $\mathrm{G}_{1}$.

configurations $x, y \in \mathbb{B}^{n}$, we use $\mathrm{D}(x, y)=\left\{i \in \mathrm{V} \mid x_{i} \neq y_{i}\right\}$ and the Hamming distance $\mathrm{d}(x, y)=|\mathrm{D}(x, y)|$.

A BAN $\mathscr{N}$ whose automata set is $\mathrm{V}$ is a set of $n$ Boolean functions such that $\mathscr{N}=\left\{\mathrm{f}_{i}: \mathbb{B}^{n} \rightarrow \mathbb{B} \mid i \in \mathrm{V}\right\}$. Given $i \in \mathrm{V}, \mathrm{f}_{i}$ is called the local transition function of automaton $i$. It predetermines its behaviour depending on the states of other automata that influence it and that appear consequently as literals in the Boolean expression of $\mathrm{f}_{i}$. More precisely, it predetermines its behaviour for every configuration $x \in \mathbb{B}^{n}$, meaning that if $i$ is updated in $x$, its state switches from $x_{i}$ to $\mathrm{f}_{i}(x)$. We introduce now the sign of an interaction (i.e. an influence) from $j$ to $i$, both in $\mathrm{V}$, in configuration $x \in \mathbb{B}^{n}$ with:

$$
\operatorname{sign}_{x}(j, i)=\mathrm{s}\left(x_{j}\right) \cdot\left(\mathrm{f}_{i}(x)-\mathrm{f}_{i}\left(\bar{x}^{j}\right)\right) .
$$

From this, the set of interactions that are effective in $x$ is defined as $\mathrm{A}(x)=$ $\left\{(j, i) \in \mathrm{V}^{2} \mid \operatorname{sign}_{x}(j, i) \neq 0\right\}$. And we derive directly the interaction graph, or architecture, of $\mathscr{N}$ by defining the digraph $\mathrm{G}=(\mathrm{V}, \mathrm{A})$, where $\mathrm{A}=\bigcup_{x \in \mathbb{B}^{n}} \mathrm{~A}(x)$ is the set of interactions (cf. Figure 1) and $|A|=m$. We add that automata of $\mathrm{V}$ that influence $i$ are called the neighbours of $i$, and that the neighbourhood of $i \in \mathrm{V}$ in $\mathrm{G}$ is denoted by $\mathrm{V}^{-}(i)$. In this paper, we consider BANs whose interaction graphs are simple, i.e. if there exists $(j, i) \in \mathrm{A}$, it is unique and such that $\forall x \in \mathbb{B}^{n}, \operatorname{sign}_{x}(j, i) \neq 0$ and is constant, and thus denoted simply by $\operatorname{sign}(j, i) \in \mathbb{1}$. Remark that if $\operatorname{sign}(j, i)=+1$ (resp. $\operatorname{sign}(j, i)=-1),(j, i)$ is an activating (resp. inhibiting) interaction so that the state of $i$ tends to mimic (resp. negate) that of $j$. The digraph obtained by labelling each arc $(i, j) \in \mathrm{A}$ with $\operatorname{sign}(i, j)$ is the signed interaction graph of $\mathscr{N}$. We also denote it by $\mathrm{G}$, in order not to burden the reading. To finish on BANs, we add that the sign of a path in $G$ equals the product of the signs of its arcs, which leads us to define positive and negative cycles in $\mathrm{G}$. Abusing notations, a cycle $\mathrm{C}$ of $\mathrm{G}$ is positive (resp. negative) if $\operatorname{sign}(\mathrm{C})=+1$ (resp. $\operatorname{sign}(\mathrm{C})=-1)$. Note that this paper only deals with BANs whose signed interaction graphs do not contain any negative cycle.

\subsection{Transition graphs}

In a BAN $\mathscr{N}$, we call elementary transition a couple of configurations $(x, y) \in$ $\mathbb{B}^{n} \times \mathbb{B}^{n}$, such that $y$ is obtained by updating automata of $x$, meaning that $\exists \mathrm{W} \neq \emptyset \subseteq \mathrm{V}, \forall i \in \mathrm{V}, y_{i}=x_{i}$ if $i \in \mathrm{V} \backslash \mathrm{W}$ and $y_{i}=\mathrm{f}_{i}(x)$ if $i \in \mathrm{W}$. If $x=y$ (i.e. 


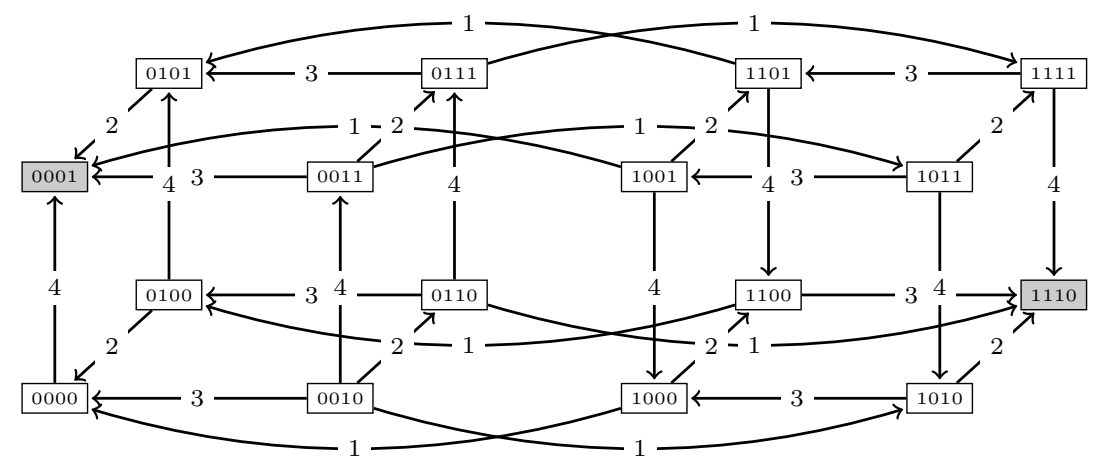

Fig. 2. The asynchronous transition graph $\mathscr{G}_{1}$ of $\mathscr{N}_{1}$, defined in Figure 1 , and its two attractors, the stable configurations 0001 and 1110 (ineffective transitions have been omitted).

$\mathrm{d}(x, y)=0)$, then transition $(x, y)$ is said to be ineffective. Conversely, if $0<$ $\mathrm{d}(x, y) \leq n$, it is effective. As evoked, a transition $(x, y)$ can induce the updating of automata of a subset $W \neq \emptyset \subseteq V$. If $|W|>1,(x, y)$ is synchronous. Otherwise, if $|W|=1,(x, y)$ is asynchronous and is denoted by $x \longrightarrow y$, which implies that $\mathrm{d}(x, y) \leq 1$. Let $\mathrm{T}=\left\{x \longrightarrow y \mid x, y \in \mathbb{B}^{n}\right\}$ be the set of asynchronous transitions of $\mathscr{N}$. Digraph $\mathscr{G}=\left(\mathbb{B}^{n}, \mathrm{~T}\right)$ is then the asynchronous transition graph of $\mathscr{N}$ (cf. Figure 2). In other words, $\mathscr{G}$ represents the discrete dynamical system associated to $\mathscr{N}$ when the latter is governed by the non-deterministic "perfectly" asynchronous updating mode. In this paper, as said before, results presented are for BANs subjected to this specific updating mode. However, unless we mention it, they trivially extend to the elementary (also called general) updating mode, that considers both synchronous and asynchronous transitions [16].

Let $\mathscr{N}$ be an arbitrary BAN of size $n$ and let $\mathscr{G}=\left(\mathbb{B}^{n}, T\right)$ its associated asynchronous transition graph. Let $x \in \mathbb{B}^{n}$ be any configuration of $\mathscr{N}$. We define as a trajectory of $x$ any path in $\mathscr{G}$ that starts in $x$. A strongly connected component (SCC) of $\mathscr{G}$ that does not admit any outgoing transition is a terminal strongly connected component (TSCC). An attractor of $\mathscr{N}$ is a TSCC of $\mathscr{G}$, that corresponds thus to an asymptotic behaviour of $\mathscr{N}$. The size of an attractor is defined as the number of configurations it contains. An attractor of size 1 (resp. of size greater than 1) is a stable configuration (resp. a stable oscillation). Finally, we call convergence time of a configuration $x$ the length of the shortest trajectory that leads it to an attractor and convergence time of a BAN the biggest convergence time of all configurations in $\mathbb{B}^{n}$.

\section{Results}

This section aims at presenting the main results obtained on BANs with no negative cycles: a relationship with the Boolean monotonicity, a new simple and 
neat proof of the Thomas' second rule and a result about the linear convergence time of such BANs depending on their sizes.

\subsection{Negative cycles and monotonicity}

The global transition function of a BAN $\mathscr{N}=\left\{\mathrm{f}_{i}: \mathbb{B}^{n} \rightarrow \mathbb{B} \mid i \in \mathrm{V}\right\}$ is the map $\mathrm{f}: \mathbb{B}^{n} \rightarrow \mathbb{B}^{n}$ such that, for all $x \in \mathbb{B}^{n}$ and $i \in \mathrm{V}, \mathrm{f}(x)_{i}=\mathrm{f}_{i}(x)$. We say that $\mathscr{N}$ is monotone if

$$
\forall x, y \in \mathbb{B}^{n}, x \leq y \Longrightarrow \mathrm{f}(x) \leq \mathrm{f}(y),
$$

where $\leq$ is the usual partial order on $\mathbb{B}^{n}$. Equivalently, $\mathscr{N}$ is monotone if its signed interaction graph has only positive arcs. For all $\mathrm{W} \subseteq \mathrm{V}$, the $W$-switch of $\mathscr{N}$ is the BAN $\mathscr{N}^{\mathrm{W}}=\left\{\mathrm{f}_{i}^{\mathrm{W}}: \mathbb{B}^{n} \rightarrow \mathbb{B} \mid i \in \mathrm{V}\right\}$ such that

$$
\forall x \in \mathbb{B}^{n}, \mathrm{f}^{\mathrm{W}}(x)=\overline{\mathrm{f}\left(\bar{x}^{\mathrm{W}}\right)} \mathrm{W} .
$$

In other words, $\mathscr{N}^{\mathrm{W}}$ is obtained from $\mathscr{N}$ by replacing, for automata in $\mathrm{W}$, ones by zeros and zeros by ones. By doing this operation, a lot of properties are preserved. In particular, the asynchronous transition graph of $\mathscr{N}^{\mathrm{W}}$ is isomorphic to that of $\mathscr{N}$ (the isomorphism being $x \mapsto \bar{x}^{\mathrm{W}}$ ). Besides, the signed interaction graph $\mathrm{G}^{\mathrm{W}}$ of $\mathscr{N}^{\mathrm{W}}$ is obtained from the signed interaction graph $\mathrm{G}$ of $\mathscr{N}$ by: (i) kipping exactly the same vertices and the same arcs, and (ii) changing the sign of ingoing and outgoing $\operatorname{arcs}$ of $\mathrm{W}$, i.e. $\operatorname{arcs}(j, i)$ such that $j \in \mathrm{W}$ and $i \notin \mathrm{W}$ and $\operatorname{arcs}(j, i)$ such that $j \notin \mathrm{W}$ and $i \in \mathrm{W}$. So $\mathrm{G}$ and $\mathrm{G}^{\mathrm{W}}$ have the same cycles, and these cycles have the same signs, even if the repartition of signs on arcs may differ. These similarities lead us to say that a BAN $\mathscr{N}^{\prime}$ is equivalent to $\mathscr{N}$ if $\mathscr{N}^{\prime}$ is the $\mathrm{W}$-switch of $\mathscr{N}$ for some $\mathrm{W} \subseteq \mathrm{V}$ (cf. Figure 3).

Proposition 1. Let $\mathscr{N}$ be a BAN and let $G$ be its signed interaction graph. If $G$ is strongly connected and has no negative cycles, then $\mathscr{N}$ is equivalent to a monotone BAN.

Proof. The proof is based on arguments that we can find in $[1,8]$. First of all, let us note that if $\mathrm{G}$ has no negative arcs, it is monotone by definition. So, let us admit that $\mathrm{G}$ has negative arcs.

Pick any vertex $i \in \mathrm{V}$ of $\mathrm{G}$. For any vertex $j \in \mathrm{V}, \mathrm{G}$ has at least one path from $i$ to $j$ (since $\mathrm{G}$ is strongly connected), and all paths from $i$ to $j$ have the same sign (since otherwise $\mathrm{G}$ would have a negative cycle). Let us denote by $\operatorname{sign}_{i j} \in 11$ this sign, and let $\mathrm{W}$ be the set of vertices $j$ such that $\operatorname{sign}_{i j}=+1$. We shall prove that an arc $(k, l) \in \mathrm{A}$ of $\mathrm{G}$ is negative if and only if it is entering or leaving $\mathrm{W}$. Let $(k, l)$ be any arc of $\mathrm{G}$.

If $k, l \in \mathrm{W}$ then $\mathrm{G}$ has a positive path from $i$ to $k$ and from $i$ to $l$. Thus, if $(k, l)$ is negative, then together with the positive path from $i$ to $k$, it gives a negative path from $i$ to $l$, which is a contradiction. Thus $(k, l)$ is positive, and we prove in a similar way that $(k, l)$ is positive if $k, l \notin \mathrm{W}$.

If $(k, l)$ is an outgoing arc of $\mathrm{W}(k \in \mathrm{W}$ and $l \notin \mathrm{W})$ then $\mathrm{G}$ has a positive 


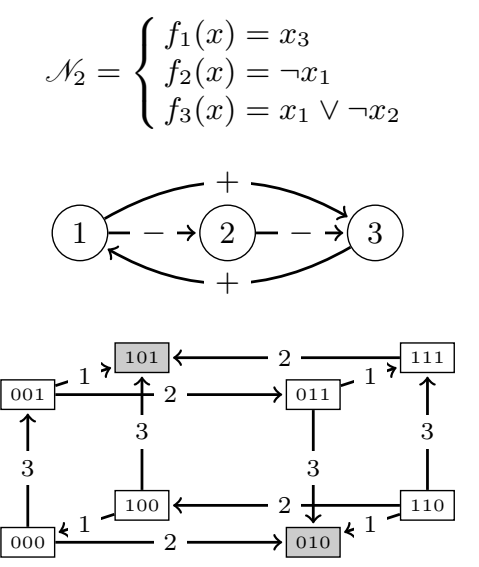

(a)

$$
\mathscr{N}_{2}{ }^{\{2\}}=\left\{\begin{array}{l}
f_{1}(x)=x_{3} \\
f_{2}(x)=x_{1} \\
f_{3}(x)=x_{1} \vee x_{2}
\end{array}\right.
$$
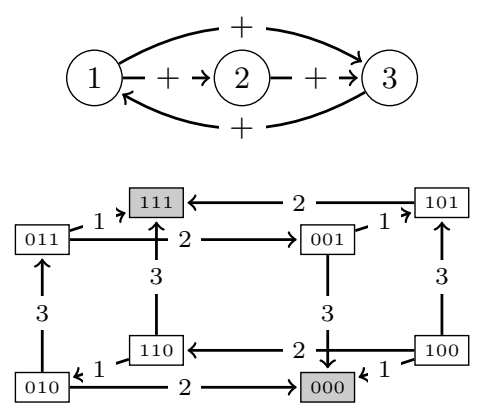

(b)

Fig. 3. (a) A BAN $\mathscr{N}_{2}$ of size 3 , its signed interaction graph $\mathrm{G}_{2}$ and its asynchronous transition graph $\mathscr{G}_{2}$; (b) the monotone BAN $\mathscr{N}_{2}{ }^{\{2\}}$ equivalent to $\mathscr{N}_{2}$ (its $\{2\}$-switch actually) and its associated asynchronous transition graph $\mathscr{G}_{2}^{\{2\}}$.

path from $i$ to $k$ and a negative path from $i$ to $l$. Thus if $(k, l)$ is positive, then together with the positive path from $i$ to $k$ it gives a positive path from $i$ to $l$, which is a contradiction. Thus $(k, l)$ is negative, and we prove in a similar way that $(k, l)$ is negative if it is an ingoing arc of $\mathrm{W}$.

Hence, an arc of $\mathrm{G}$ is negative if and only if it is an ingoing or an outgoing arc of W. Let $\mathscr{N}^{\mathrm{W}}$ be the $\mathrm{W}$-switch of $\mathscr{N}$, and let $\mathrm{G}^{\mathrm{W}}$ be its signed interaction graph. As said above, $\mathrm{G}^{\mathrm{W}}$ is obtained from $\mathrm{G}$ by changing the signs of ingoing and outgoing $\operatorname{arcs}$ of $\mathrm{W}$. We deduce that $\mathrm{G}^{\mathrm{W}}$ has only positive $\operatorname{arcs}$, so that $\mathscr{N}^{\mathrm{W}}$ is monotone (cf. Figure 3).

Actually, it is possible to characterise BANs that are equivalent to a monotone BAN in terms of undirected negative cycle. To do so, we need the following characterisation of Harary [8]: An arc-signed undirected graph has no negative cycles if and only if there exists a set of vertices $W$ such that an edge of the graph is negative if and only if it has one end in $W$ and another outside $W$. Now, let $\mathscr{N}$ be a BAN with signed interaction graph $G$, and let $\mathrm{U}(\mathrm{G})$ be the undirected arc-signed graph obtained from $G$ by forgetting directions $(\mathrm{U}(\mathrm{G})$ may have loops, and $U(G)$ has both a positive arc and a negative arc between two vertices if $G$ has a negative cycle of length two between these vertices). Using the characterisation of Harary, we can obtain the following characterisation: $A$ $B A N \mathscr{N}$ is equivalent to a monotone $B A N$ if and only if $U(G)$ has no negative cycle. The previous proposition (which is enough for our purpose) follows from the fact that if $\mathrm{G}$ is strongly connected, then $\mathrm{U}(\mathrm{G})$ has no negative cycles if and only if $G$ has no negative cycles. Analogues of this characterisation are well known in the context of differential systems, see $[9,24]$ for instance. 


\subsection{Stable configurations and stable oscillations}

In this section, we show how to recover known results about stable configurations and oscillations from the previous proposition and the following two easy results on monotone BANs.

Proposition 2. Suppose that $\mathscr{N}$ is monotone and such that, $\forall i \in V, f_{i} \neq c s t$. Then, configurations $0 \ldots 0$ and $1 \ldots 1$ are stable configurations.

Proof. Let $\mathrm{f}$ be the global transition function of $\mathscr{N}$. If $\mathrm{f}(0 \ldots 0) \neq 0 \ldots 0$ then $\mathrm{f}_{i}(0 \ldots 0)=1$ for some $i \in \mathrm{V}$. Let $x \in \mathbb{B}^{n}$. Since $0 \ldots 0 \leq x$ and $\mathscr{N}$ is monotone, we have $\mathrm{f}_{i}(0 \ldots 0) \leq \mathrm{f}_{i}(x)$ so $\mathrm{f}_{i}(x)=1$. Thus $\mathrm{f}_{i}(\cdot)=$ cst $=1$, which is a contradiction. As a consequence, $f(0 \ldots 0)=0 \ldots 0$ and configuration $0 \ldots 0$ is stable. We prove in a similar way that $\mathrm{f}(1 \ldots 1)=1 \ldots 1$.

Proposition 3. Suppose that $\mathscr{N}$ is monotone. For all $x \in \mathbb{B}^{n}$, the asynchronous transition graph of $\mathscr{N}$ has a path of length at most 2 from $x$ to a stable configuration.

Proof. Let $\mathscr{G}$ be the asynchronous transition graph of $\mathscr{N}$ whose global transition function is $\mathrm{f}$. Let $\mathrm{P}=x^{0} x^{1} \ldots x^{p}$ be a decreasing path of $\mathscr{G}$ starting from $x=x^{0}$, and of maximal length for this property, i.e.

$$
x^{0} \geq x^{1} \geq \cdots \geq x^{p} \quad \text { and } \quad x^{p} \leq \mathrm{f}\left(x^{p}\right) .
$$

Let $\mathrm{Q}=y^{0} y^{1} \ldots y^{q}$ be an increasing path of $\mathscr{G}$ starting from $y^{0}=x^{p}$, and of maximal length for this property, i.e.

$$
y^{0} \leq y^{1} \leq \cdots \leq y^{q} \quad \text { and } \quad y^{q} \geq \mathrm{f}\left(y^{q}\right) .
$$

Let $k<q$. If $y^{k} \leq f\left(y^{k}\right)$ then $y^{k} \leq y^{k+1} \leq \mathrm{f}\left(y^{k}\right)$, and since $\mathscr{N}$ is monotone, $\mathrm{f}\left(y^{k}\right) \leq \mathrm{f}\left(y^{k+1}\right)$. Thus:

$$
y^{k} \leq \mathrm{f}\left(y^{k}\right) \Longrightarrow y^{k+1} \leq \mathrm{f}\left(y^{k+1}\right) .
$$

Since $y^{0}=x^{p}$, we have $y^{0} \leq \mathrm{f}\left(y^{0}\right)$ and we deduce that $\forall 0 \leq k \leq q, y^{k} \leq \mathrm{f}\left(y^{k+1}\right)$. In particular, $y^{q} \leq \mathrm{f}\left(y^{q}\right)$, and we deduce that $y^{q}=\mathrm{f}\left(y^{q}\right)$. Thus the concatenation of $\mathrm{P}$ and $\mathrm{Q}$ gives a path from $x$ to the stable configuration $y^{q}$. Since $\mathrm{P}$ and $\mathrm{Q}$ are decreasing and increasing, we have $p, q \leq n$. As a consequence, the concatenation of $\mathrm{P}$ and $\mathrm{Q}$ is of length at most $2 n$.

Let $\mathscr{N}$ be BAN and let G be its signed interaction graph. Aracena [1] (see also [2]) proved the following: If $G$ is strongly connected and has no negative cycles (and at least one arc) then the global transition function has at least two stable configurations. This is an immediate consequence of Propositions 1 and 2. Actually, all the arguments of the original proof are more or less contained in the proof of these two propositions, so we cannot speak about a proof simplification here. However, the interest in Proposition 1, which gives a new relationship 
between monotonicity and negative cycles, is clearly visible with the following second application.

In [18], the following discrete version of the second Thomas' rule has been established, with a quite complex proof: If $G$ has no negative cycles, then the asynchronous transition graph of $\mathscr{N}$ has no stable oscillations. Actually, a very easy proof results directly from Propositions 1 and 3. Indeed, suppose that G has no negative cycles, and suppose first that $\mathrm{G}$ is strongly connected. Then $\mathscr{N}$ is equivalent to a monotone BAN $\mathscr{N}^{\prime}$ by Proposition 1 . And we deduce from Proposition 3 that, in the asynchronous transition graph $\mathscr{G}^{\prime}$ of $\mathscr{N}^{\prime}$, a stable configuration can be reached from every initial configuration. Since the asynchronous transition graph $\mathscr{G}$ of $\mathscr{N}$ is isomorphic to $\mathscr{G}^{\prime}$, we have proven the following: If $G$ has no negative cycles and is strongly connected, then, in $\mathscr{G}$, a stable configuration can be reached from any initial configuration (*). Now, suppose that G is not strongly connected. Then, by applying $(*)$ on the SCCs of G, proceeding from the initial ones to the terminal ones (according to the underlying topological ordering of the SCCs), we obtain the same conclusion: a stable configuration can be reached from any initial configuration. As a consequence, $\mathscr{G}$ has no stable oscillations, which had to be proven.

\subsection{More precisions about convergence times}

In this part, for a given monotone BAN $\mathscr{N}$ of size $n$ and an arbitrary initial configuration $x \in \mathbb{B}^{n}$, we interest in the set $\mathscr{A}(x)$ of stable configurations reachable from $x$. We will show the followings results:

- there exist two stables configurations $a^{+}(x)$ and $a^{-}(x)$ of $\mathscr{A}(x)$ such that for any $a \in \mathscr{A}(x), a^{-}(x) \leq a \leq a^{+}(x)$;

$-a^{-}(x)$ and $a^{+}(x)$ are reachable from $x$ in less than $2 n-4$ transitions and, in some cases, this bound is tight;

- there exists a configuration $a \in \mathscr{A}(x)$ such that $a$ is reachable from $x$ in at most $n$ transitions.

To compute $a^{-}(x)$, we will proceed as in the proof of Proposition 3, by using a decreasing path from $x$ of maximal length and, then, an increasing path of maximal length. The computation of $a^{+}(x)$ is done symmetrically. Let us denote by $\mathbf{0}(x)$ (resp. $\mathbf{1}(x)$ ) a configuration (which can be proven to be unique) resulting from following a decreasing path $\mathrm{P}$ of maximal length (resp. an increasing path $\mathrm{Q}$ of maximal length).

Lemma 1. Let $\mathscr{N}$ be a monotone $B A N$ and $\mathscr{G}$ its associated asynchronous transition graph. Consider a configuration $x \in \mathbb{B}^{n}$ of $\mathscr{N}$. If there exists an automaton $i$ such that $\mathbf{0}(x)_{i}=1$, then the configurations where the state of automaton $i$ equals 0 are not reachable from $x$. Conversely, if there exists an automaton $i$ such that $\mathbf{1}(x)_{i}=0$, the configurations where the state of automaton $i$ equals 1 are not reachable from $x$. 
Proof. Let $x \in \mathbb{B}^{n}$. First, let us prove the first part of the lemma. Consider an automaton $i$ such that $\mathbf{0}(x)_{i}=1$, and let us suppose that there exists a configuration $y \in \mathbb{B}^{n}$ reachable from $x$ such that $\mathrm{f}_{i}(y)=0$. Without loss of generality, we suppose that $y$ is the closest configuration to $x$ with an automaton $k$ such that $\mathrm{f}_{k}(y)=0$ and $\mathbf{0}(x)_{k}=1$ (in $\left.\mathscr{G}\right)$. Since $\mathrm{f}_{i}(y)=0, \mathrm{f}_{i}(\mathbf{0}(x))=1$ and $\mathrm{f}_{i}$ is monotone, there exists at least one automaton $j \in V^{-}(i)$ such that $y_{j}=0$ and $\mathbf{0}(x)_{j}=1$. Now, since $\mathbf{0}(x)_{j}=1$, we have $x_{j}=1$ and thus $j$ switches from state 1 to state 0 along the path from $x$ to $y$, which is a contradiction with the fact that $y$ is the closest configuration to $x$ with an automaton $k$ such that $\mathrm{f}_{k}(y)=0$ and $\mathbf{0}(x)_{k}=1$. We prove in a similar way the second part of the lemma.

Corollary 1. Let $\mathscr{N}$ be a monotone $B A N$ and $\mathscr{G}$ its associated asynchronous transition graph. Consider a configuration $x \in \mathbb{B}^{n}$ of $\mathscr{N}$. For any configuration $y$ reachable from $x, \mathbf{0}(x) \leq \mathbf{0}(y)$.

Lemma 2 below gives a mean to detect irreversible transitions, i.e. transitions that make an automaton stable in the sense that its state cannot change anymore.

Lemma 2. Let $\mathscr{N}$ be a monotone $B A N$ and $\mathscr{G}$ its associated asynchronous transition graph. Consider a configuration $x \in \mathbb{B}^{n}$ of $\mathscr{N}$. If there exists an automaton $i \in V$ such that $x_{i}=0$ and $f_{i}(\mathbf{0}(x))=1$, configurations with automaton $i$ in state 0 are not reachable from a configuration reachable by $x$ with automaton $i$ in state 1.

Proof. Consider a configuration $x \in \mathbb{B}^{n}$ of $\mathscr{N}$ with an automaton $i \in \mathrm{V}$ such that $x_{i}=0$ and $\mathrm{f}_{i}(\mathbf{O}(x))=1$. Let $y \in \mathbb{B}^{n}$ be a configuration with $y_{i}=1$ that is reachable from $x$, and $z \in \mathbb{B}^{n}$ a configuration reachable from $y$. By Corollary $1, \mathbf{0}(x) \leq \mathbf{0}(y)$ and $\mathrm{f}_{i}(\mathbf{0}(y))=1$. Configuration $\mathbf{0}(y)$ is reachable from $y$ by a decreasing path and, since $\mathrm{f}_{i}$ is monotone, $\mathbf{0}(y)=1$. By Corollary 1 , $\mathbf{0}(y) \leq \mathbf{0}(z) \leq z$ and, necessarily, $z_{i}=1$.

Let us denote by $a^{-}(x)\left(\right.$ resp. $\left.a^{+}(x)\right)$ the configuration $\mathbf{1}(\mathbf{0}(x))(\operatorname{resp} . \mathbf{0}(\mathbf{1}(x)))$. Note that both these configurations are reachable from $x$.

Theorem 1. Let $\mathscr{N}$ be a monotone BAN of size $n$ and $\mathscr{G}$ its associated asynchronous transition graph. Consider an arbitrary configuration $x \in \mathbb{B}^{n}$ of $\mathscr{N}$. Configurations $a^{-}(x)$ and $a^{+}(x)$ are stable and $\forall a \in \mathscr{A}(x), a^{-}(x) \leq a \leq a^{+}(x)$.

Proof. From the proof of Proposition $3, a^{-}(x)$ is stable. Symmetrically, it is easy to show that $a^{+}(x)$ is too.

Consider any $a \in \mathscr{A}(x)$. Since $a$ is reachable from $x$, by Lemma 1 we have $\mathbf{0}(x) \leq$ $a$. Now, consider a minimal increasing path from $\mathbf{0}(x)$ to $a^{-}(x)$. Suppose (for a contradiction) that there is an automaton $i \in \mathrm{V}$ such that $a_{i}=0$ and $a^{-}(x)_{i}=1$. Without loss of generality, we suppose that $i$ is the first that is updated along the path with this property $(*)$. Then, let $y \in \mathbb{B}^{n}$ be the configuration reached from $\mathbf{0}(x)$ just before the updating of $i$. Thus we have $\mathrm{f}_{i}(y)=1$. Since $a \geq \mathbf{0}(x)$ and since $i$ respects $(*), a \geq y$ holds. Moreover, since $\mathrm{f}_{i}$ is monotone, $\mathrm{f}_{i}(a)=1$, which 
is a contradiction with the fact that $a$ is a stable configuration. Thus $a^{-}(x) \leq a$ and we prove with similar arguments that $a \geq a^{+}(x)$.

Let $\mathcal{O}(\mathrm{f})$ be the time complexity for evaluating the local transition functions. Remark that the computation time to find $a^{+}(x)$ and $a^{-}(x)$ is $\mathcal{O}(n+m+\mathrm{f})$. Despite this time complexity in the general case, genetic regulation networks are known to have a connectivity (i.e. the average in-degree of G) $2 \leq \mathrm{K} \leq 3$ [12]. As a consequence, we can assume that the time complexity in real-life examples is $\mathcal{O}(n)$.

Let us now give an upper bound (only valid for the asynchronous updating mode) on the convergence time from $x$ to $a^{-}(x)$ (and conversely for $a^{+}(x)$ ), i.e. an upper bound on the length of shortest paths from $x$ to $a^{-}(x)$. More precisely, let us prove that the convergence time from $x$ to $a^{-}(x)$ is at most $2 n-4$ (for $n \geq 3$ ). Suppose first that no local update functions are constant, so that $1 \ldots 1$ and $0 \ldots 0$ are stable configurations. Consider that $x$ is not $1 \ldots 1$ (otherwise $x=a^{-}(x)$ and there is nothing to prove). If $\mathbf{0}(x)=0 \ldots 0$ then $a^{-}(x)=\mathbf{0}(x)$ and the convergence time is $d(x, 0 \ldots 0)<n$. So, suppose that $\mathbf{0}(x)$ is not $0 \ldots 0$. Then the convergence time from $x$ to $\mathbf{0}(x)$ is at most $n-2$, and the convergence time from $\mathbf{0}(x)$ to $a^{-}(x)=\mathbf{1}(\mathbf{0}(x))$ is at most $n-1$. Now, suppose (for a contradiction) that the convergence time from $x$ to $a^{-}(x)$ is $2 n-3$. Then the convergence time from $x$ to $\mathbf{0}(x)$ is $n-2$ and the convergence time from $\mathbf{0}(x)$ to $a^{-}(x)$ is $n-1$. We deduce that, in configuration $x$, there is a unique automaton, let us call it $i$, whose state is 0 , and we deduce that $a^{-}(x)=1 \ldots 1$. Since there are no transitions from $x$ to $a^{-}(x)$ (otherwise the convergence time is 1) and since the network is monotone, we deduce that for all $y \in \mathbb{B}^{n}$, if $y_{i}=0$ then $\mathrm{f}_{i}(y)=0$. But it means that configuration $1 \ldots 1$ cannot be reached from $x$, which is a contradiction. As a consequence, the convergence time from $x$ to $a^{-}(x)$ is at most $2 n-4$. Now, from this, it is easy to see that if the network contains $k$ constant local update function, then the convergence time from $x$ to $a^{-}(x)$ is at most $k+2(n-k)-4$, so that $2 n-4$ is a correct bound in every case. Now, studying the dynamics of networks defined as

$$
\left\{\begin{array}{l}
\mathrm{f}_{1}(x)=x_{1} \vee x_{n} \\
\forall i \in\{2, \ldots, n-1\}, \mathrm{f}_{i}(x)=x_{i-1} \\
\mathrm{f}_{n}(x)=x_{n-1} \wedge x_{n}
\end{array}\right.
$$

evidences that the bound is obtained (it is easy to show it with $n=3$ by computing the convergence time of $x=101$ to $\left.a^{-}(x)=110\right)$.

Also, notice that if a stable configuration $a$ verifies $a^{-}(x) \leq a \leq a^{+}(x)$, we cannot conclude that $a \in \mathscr{A}(x)$. The simplest example is the BAN of size 3 defined as

$$
\left\{\begin{array}{l}
\mathrm{f}_{1}(x)=x_{2} \wedge x_{3} \\
\forall i \in\{2,3\}, \mathrm{f}_{i}(x)=x_{i-1} \vee x_{i}
\end{array} .\right.
$$

Consider the initial configuration $x=100$. We have $a^{-}(x)=000$ and $a^{+}(x)=$ 111. Furthermore, $a^{-}(x) \leq a=001 \leq 111$ is stable but is not reachable from $x$. 
Now, we show that every configuration can converge in no more than $n$ transitions.

Theorem 2. Let $\mathscr{N}$ be a monotone BAN of size $n$ and $\mathscr{G}$ its associated asynchronous transition graph. Consider an arbitrary configuration $x \in \mathbb{B}^{n}$ of $\mathscr{N}$. There exists a stable configuration $a \in \mathscr{A}(x)$ such that a is reachable in at most $n$ transitions from $x$ without switching the state of an automaton twice.

Proof. Consider an arbitrary configuration $x \in \mathbb{B}^{n}$ of $\mathscr{N}$ and the following algorithm that returns a couple of configurations $(y, z) \in \mathbb{B}^{n} \times \mathbb{B}^{n}$ :

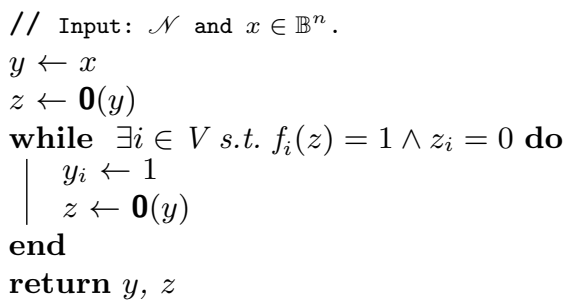

Consider an automaton $i$ such that $z_{i}=1$. Since $z=\mathbf{0}(y), \mathrm{f}_{i}(z)=1$. Now, consider an automaton $j$ such that $z_{j}=0$. Suppose that $\mathrm{f}_{j}(z)=1$. In this case, either $y_{j}=0$, which is a contradiction because the algorithm should have switched its state to 1 , or $y_{j}=1$, and, since $z=\mathbf{0}(y)$, this contradicts the monotonicity of $\mathrm{f}_{j}$. Thus, $z$ is a stable configuration.

Now, let us prove that $z$ is reachable from $x$. Consider the following invariant: When an automaton $i$ of $y$ is switched from 0 to $1, f_{i}(y)=1$. Since $y \geq \mathbf{0}(y)$, $\mathrm{f}_{i}(\mathbf{0}(y))=1$ and $\mathrm{f}_{i}$ is monotone, then $\mathrm{f}_{i}(y)=1$. Thus, $y$ is reachable from $x$. And since $z=\mathbf{0}(y), z$ is reachable from $x$.

By Lemma 2, there is a path of irreversible transitions from $x$ to $y$. Since $z=$ $\mathbf{0}(y)$, there is a decreasing path from $y$ to $z$. Thus, $z$ is reachable in no more than $n$ transitions from $x$ without switching the state of an automaton twice. And we deduce that the convergence time of $x$ is at most $n$.

\section{Conclusion and perspectives}

In this paper, we have focused on BANs without negative cycles and have shown (or given new neater proofs of) pertinent results in a context at the frontier of theoretical computer science and theoretical biology. Notice once again that, although proofs are given in the framework of BANs subjected with the asynchronous updating mode (for easing the reading), they remain valid for the much more complicated elementary updating mode. Of course, this is due to the restriction we did on BANs themselves, by considering only those with no negative cycles that are known to admit no stable oscillations as asymptotic behaviours. Rather than recalling now the results presented in the previous lines, we prefer drawing some perspectives that we believe relevant for further works.

The first perspective directly comes from the last result presented. It shows that, in a monotone BAN of size $n$ (and its equivalent BANs), any configuration 
can reach dynamically a stable configuration in at most $n$ transitions. A natural question that remains to be answered to in the same framework as that used in this paper is the following: are the stable configurations that are reachable from a given arbitrary initial configuration all reachable in $2 n-4$ transitions? If not, are they in a polynomial number of transitions according to $n$ ? Furthermore, this study allows to give, for each configuration of monotone(-equivalent) BANs, a basic representation of its set of attractors, and thus, of the attraction basins of the underlying dynamical systems. However, it should be possible to go further and give more precisions about these sets, as it has been done in the case of Minority in [21]. Also, of course, it would be interesting to characterise BANs of size $n$ inducing negative cycles in their architecture which would not break the convergence time property in at most $n$ transitions.

Moreover, following $[6,16,20]$, we know that the choice of the updating mode is crucial for the dynamical behaviour of a BAN, even a monotone one, to have certain properties. For instance, although monotone BANs admit only stable configurations as asymptotic behaviours according to both the asynchronous and elementary updating modes, that is not the case if they are subjected to the parallel updating mode. For instance, consider a monotone positive cycle of size $n$ evolving in parallel, it admits stable oscillations (or limit cycles) and, consequently, does not converge necessarily. As a consequence, it would be of interest to dive this study and its associated perspectives into the context of deterministic updating modes, such as the block-sequential ones.

Finally, on the basis of the present work, the last perspective that seems amongst the most relevant according to us would be to work on the discrete version of the monotone function theory on Banach spaces notably developed by Hirsch and Smith in $[9,24]$. This would lead us to obtain a better understanding of the common properties of such continuous and discrete objects and, possibly, to highlight fundamental properties they do not share.

Acknowledgements This work has been supported by the ANR projects BioTempo (ANR-10-BLAN-0218) and Synbiotic (ANR-10-BLAN-0307) and by Bottollier Motoculture through a working group organised in Sallanches at the beginning of 2013 and we are particularly grateful to Pascal Bottollier for having fostered us during a week.

\section{References}

1. Aracena, J.: Maximum number of fixed points in regulatory Boolean networks. Bulletin of Mathematical Biology 70, 1398-1409 (2008)

2. Aracena, J., Demongeot, J., Goles, E.: Positive and negative circuits in discrete neural networks. IEEE Transactions on Neural Networks 15, 77-83 (2004)

3. Elspas, B.: The theory of autonomous linear sequential networks. IRE Transactions on Circuit Theory 6, 45-60 (1959)

4. Goles, E.: Fixed point behavior of threshold functions on a finite set. SIAM Journal on Algebraic and Discrete Methods 3, 529-531 (1982) 
5. Goles, E., Martínez, S.: Neural and automata networks: dynamical behaviour and applications. Kluwer Academic Publishers (1990)

6. Goles, E., Salinas, L.: Sequential operator for filtering cycles in Boolean networks. Advances in Applied Mathematics 45, 346-358 (2010)

7. Golomb, S.W.: Shift register sequences. Holden-Day (1967)

8. Harary, F.: On the notion of balance of a signed graph. Michigan Mathematical Journal 2, 143-146 (1953)

9. Hirsch, M.W., Smith, H.: Monotone maps: a review. Journal of Difference Equations and Applications 11, 379-398 (2005)

10. Hopfield, J.J.: Neural networks and physical systems with emergent collective computational abilities. Proceedings of the National Academy of Sciences of the USA 79, 2554-2558 (1982)

11. Kauffman, S.A.: Metabolic stability and epigenesis in randomly constructed genetic nets. Journal of Theoretical Biology 22, 437-467 (1969)

12. Kauffman, S.A.: Origins of order: self-organization and selection in evolution. Oxford University Press (1993)

13. Kleene, S.C.: Automata studies, Annals of Mathematics Studies, vol. 34, chap. Representation of events in nerve nets and finite automata, pp. 3-41. Princeton Universtity Press (1956)

14. McCulloch, W.S., Pitts, W.H.: A logical calculus of the ideas immanent in nervous activity. Bulletin of Mathematical Biophysics 5, 115-133 (1943)

15. von Neumann, J.: Theory of self-reproducing automata. University of Illinois Press (1966)

16. Noual, M.: Updating automata networks. Ph.D. thesis, cole normale suprieure de Lyon (2012), http://tel.archives-ouvertes.fr/tel-00726560

17. Remy, É., Ruet, P., Thieffry, D.: Graphic requirement for multistability and attractive cycles in a Boolean dynamical framework. Advances in Applied Mathematics 41, 335-350 (2008)

18. Richard, A.: Negative circuits and sustained oscillations in asynchronous automata networks. Advances in Applied Mathematics 44, 378-392 (2010)

19. Richard, A., Comet, J.P.: Necessary conditions for multistationarity in discrete dynamical systems. Discrete Applied Mathematics 155, 2403-2413 (2007)

20. Robert, F.: Discrete iterations: a metric study. Springer Verlag (1986)

21. Rouquier, J.B., Regnault, D., Thierry, É.: Stochastic minority on graphs. Theoretical Computer Science 412, 3947-3963 (2011)

22. Saint Savage, N.: The effects of state dependent and state independent probabilistic updating on Boolean network dynamics. Ph.D. thesis, University of Manchester (2005)

23. Smith, A.R.: Simple computation-universal cellular spaces. Journal of the ACM 18, 339-353 (1971)

24. Smith, H.L.: Monotone dynamical systems: an introduction to the theory of competitive and cooperative systems, Mathematical surveys and monographs, vol. 41. American Mathematical Society (1995)

25. Thomas, R.: Boolean formalization of genetic control circuits. Journal of Theoretical Biology 42, 563-585 (1973)

26. Thomas, R.: On the relation between the logical structure of systems and their ability to generate multiple steady states or sustained oscillations. In: Numerical methods in the study of critical phenomena, Springer Series in Synergetics, vol. 9, pp. 180-193. Springer-Verlag (1981)

27. Thomas, R.: Regulatory networks seen as asynchronous automata: a logical description. Journal of Theoretical Biology 153, 1-23 (1991) 